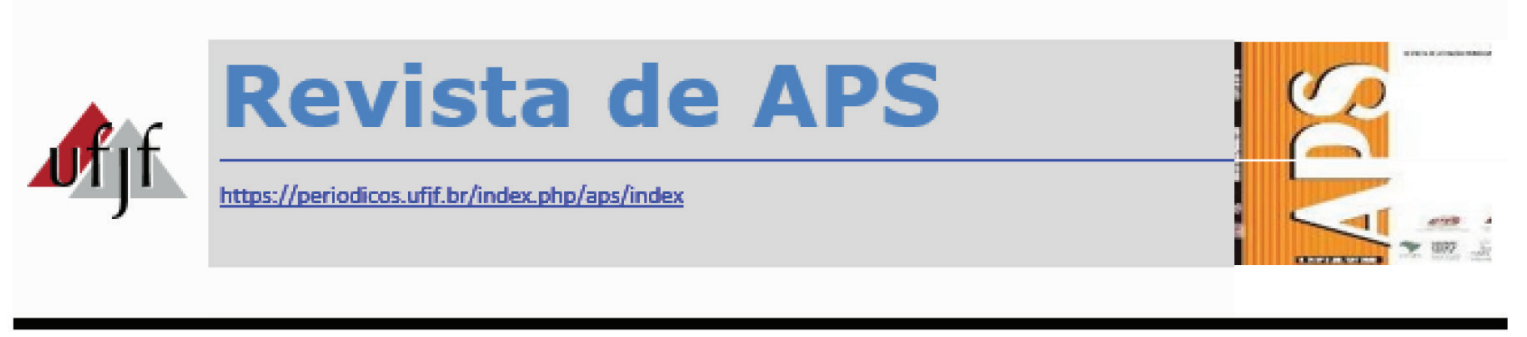

\title{
Conhecimento dos profissionais de saúde bucal sobre o processamento de produtos para saúde
}

\author{
Knowledge of oral health professionals about the processing of health \\ products
}

\author{
Darlyani Mariano da Silva ${ }^{1}$, Ana Paula Mhirdaui Sanches ${ }^{2}$, Camila Eugenia Roseira ${ }^{3}$, \\ Rosely Moralez de Figueiredo ${ }^{4}$, Silvia Carla da Silva André ${ }^{5}$
}

\begin{abstract}
RESUMO
Objetivo: avaliar o conhecimento da equipe de saúde bucal sobre o processamento de produtos para a saúde na Atenção Primária à Saúde. Material e Métodos: estudo exploratório-descritivo de abordagem quantitativa, realizado por meio da aplicação de um questionário estruturado. Dos 74 profissionais de saúde bucal alocados nas unidades, 43 participaram da pesquisa, sendo 22 auxiliares de saúde bucal e 21 odontólogos. Resultados: identificou-se que há lacunas no conhecimento da equipe quanto ao processo de esterilização (particularmente no que se refere aos recursos materiais), bem como o uso de indicadores para o monitoramento do processo. $\mathrm{O}$ uso de papel Kraft para embalagens foi apontado como correto por $16(37,20 \%)$ dos entrevistados. A ausência de informações impressas disponíveis nas unidades foi apontada por 35 (81,39\%) dos profissionais. Conclusão: Os resultados do presente estudo indicam que os profissionais de saúde bucal entrevistados ainda possuem lacunas de conhecimentos sobre o processo de esterilização, bem como sobre a definição e uso do monitoramento através de indicadores de esterilização.
\end{abstract}

PALAVRAS-CHAVE: Esterilização. Odontologia. Controle de infecções. Conhecimento.

\footnotetext{
${ }^{1}$ Graduanda em Enfermagem. Universidade Federal de São Carlos. E-mail: darlyufscar@yahoo.com.br

${ }^{2}$ Enfermeira. Residente em Oncologia no AC Camargo Câncer Center, Brasil.

${ }^{3}$ Enfermeira. Mestra. Doutoranda em Ciências da Saúde. Programa de Pós-graduação em Enfermagem. Centro de Ciências Biológicas e da Saúde. Universidade Federal de São Carlos, UFSCar, São Carlos-SP, Brasil.

${ }^{4}$ Enfermeira. Doutora. Professora Associada. Departamento de Enfermagem. Universidade Federal de São Carlos, UFSCar, São Carlos-SP, Brasil.

${ }^{5}$ Enfermeira. Doutora. Professora Adjunta. Departamento de Enfermagem. Universidade Federal de São Carlos, UFSCar, São Carlos-SP, Brasil.
} 


\begin{abstract}
Objective: To evaluate the knowledge of oral health professionals about the processing of health products in primary health care. Methods: It was used a descriptive exploratory study of quantitative approach, carried out through the application of a structured questionnaire. Of the 74 dental professionals allocated to the units, 43 participated in the survey, 22 dental assistants and 21 dentists. Results: it was found that there are gaps in staff knowledge about the sterilization process, particularly regarding material resources, as well as the use of indicators for monitoring the process. The Kraft paper used for packaging was appointed as correct for $16(37.20 \%)$ of the respondents. The lack of printed information available in the units was reported by $35(81.39 \%)$ of the professionals. Conclusion: The results of this study indicate that the interviewed oral health professionals still have knowledge gaps in the sterilization process, as well as in the definition and use of monitoring through sterilization indicators.
\end{abstract}

KEYWORDS: Sterilization. Dentistry. Infection Control. Knowledge.

\title{
INTRODUÇÃO
}

A Atenção Primária à Saúde (APS) integra um conjunto de ações individuais e coletivas disponíveis para "a promoção da saúde, a prevenção de agravos, o diagnóstico, o tratamento, a reabilitação e a manutenção da saúde". No Brasil, o sistema de Saúde é organizado em três níveis de assistência, sendo a APS considerada o primeiro destes e composta por Unidade Básica de Saúde (UBS) e Unidade de Saúde de Família (USF). Esses serviços se caracterizam pela organização do trabalho multidisciplinar e interdisciplinar, incluindo os profissionais de saúde bucal, responsáveis pela saúde bucal, que integram essas equipes. ${ }^{1}$

As atividades desempenhadas pelos profissionais de saúde bucal também possuem riscos associados de exposição a doenças como Hepatite $B$, Hepatite C e HIV. ${ }^{2}$ Assim, o uso de medidas efetivas de controle de infecção e a adesão às precauçõespadrão pela equipe de saúde bucal são essenciais para a prevenção de infecção.

Sabe-se que um dos pilares do controle e prevenção das Infecções Relacionadas à Assistência à Saúde (IRAS) está relacionado com a qualidade do processamento de produtos para a saúde, pois as infecções podem ser adquiridas em decorrência de um processamento inadequado. ${ }^{3-4}$ Ressalta-se que os próprios cirurgiões-dentistas possuem autonomia para determinar tais rotinas de trabalho, bem como a coordenação e implementação destas. ${ }^{5}$

A possibilidade de inadequações em alguma das etapas do processamento de produtos implica risco em exposição a material biológico, tanto ao cliente como para os profissionais envolvidos. Estudo realizado no estado de São Paulo mostrou que $52,4 \%$ dos produtos para saúde processados nas UBS e USF estudadas eram de saúde bucal. ${ }^{6}$ Portanto, entende-se a necessidade de esses profissionais terem 
conhecimentos sobre as boas práticas de prevenção de infecção, particularmente sobre o processamento de produtos.

Entende-se que tanto a equipe profissional quanto os pacientes estão expostos, igualmente, a uma grande variedade de agentes infecciosos. A transmissão de microrganismos pode ocorrer por diferentes vias, como por contato direto com lesões infecciosas, sangue ou saliva contaminada, além de contato indireto, mediante transferência de microrganismos presentes em um objeto contaminado, como respingos de sangue, saliva ou líquido proveniente da nasofaringe, diretamente em lesões de pele, mucosa e aerossóis. ${ }^{7}$

Ainda, falhas no processo de esterilização de produtos para a saúde utilizados pela equipe de saúde bucal, a utilização de estufas e soluções químicas para a esterilização, a não utilização de indicadores de processo, além da ausência de conhecimento sobre o processamento de produtos para saúde tem sido relatado pela literatura científica..$^{8-9}$

Estudo realizado na Universidade Federal da Paraíba (UFPB) teve como objetivo avaliar o comportamento de acadêmicos de saúde bucal com relação ao controle de infecção cruzada e mostrou que $94 \%$ dos estudantes negligenciavam a etapa de desinfecção de pré-lavagem de produtos para saúde. Os autores ressaltaram a importância de a desinfecção ser realizada antes do processo de lavagem, com o intuito de diminuir o risco de infecção. ${ }^{10}$

A responsabilidade pelo controle de infecções faz com que o processamento de produtos para saúde assuma posição de destaque, sendo necessário investimento na qualificação, pesquisa nos serviços de saúde, além de melhoria dos padrões assistenciais.

Considerando-se a importância das ações de prevenção de IRAS na APS, este estudo objetiva identificar o conhecimento da equipe de saúde bucal sobre o processamento de produtos para saúde em UBS e USF do município de São Carlos-SP.

\section{MATERIAL E MÉTODOS}

Trata-se de uma pesquisa exploratória-descritiva e de abordagem quantitativa. Este estudo foi realizado em todas as 12 Unidades Básicas de Saúde (UBS) e 17 Unidades Saúde da Família (USF) do município de São Carlos-SP.

Todos os 74 profissionais que compunham a equipe de saúde bucal da APS do município de São Carlos foram convidados a participar do estudo, dos quais 43 profissionais entre cirurgiões-dentistas e técnicos e auxiliares de saúde bucal atenderam a essa solicitação. Não participaram do estudo 31 profissionais por estarem de férias, licença médica ou recusa. Os critérios de inclusão foram: ser profissional de saúde bucal e aceitar participar da pesquisa por meio do Termo de Consentimento Livre e Esclarecido (TCLE). 
Para a coleta de dados, foi aplicado um questionário para ambas as categorias contendo cinco questões de múltipla escolha, que foi respondido na presença do pesquisador. O questionário foi baseado na Resolução da Diretoria Colegiada № 15/2012 (RDC no15) que dispõe sobre os requisitos de boas práticas para o processamento de produtos para saúde ${ }^{11}$ e avaliado por três especialistas da área, que não indicaram alterações.

Os dados foram, duplamente, digitados em planilha do programa Microsoft Office Excel $2010^{\circledR}$ para comparação de valores e identificação de possíveis erros e posteriormente analisados por meio da estatística descritiva, realizada com auxílio do software Statistical Package for the Social Sciences (SPSS) versão 22. Ressalta-se que foram analisadas e apresentadas nos resultados somente as respostas assinaladas como corretas pelos participantes.

O estudo foi aprovado pelo Comitê de Ética em Pesquisa em Seres Humanos da Universidade Federal de São Carlos (Parecer no 311.164) e todos os participantes da pesquisa assinaram o Termo de Consentimento Livre e Esclarecido (TCLE) antes de responderem ao instrumento.

\section{RESULTADOS}

Dentre os 43 membros da equipe de saúde bucal que aceitaram participar, 22 $(51,16 \%)$ eram auxiliares de saúde bucal e 21 (48,83\%) eram odontólogos. No que se refere ao tempo de trabalho nas unidades de saúde, nove $(40,90 \%)$ auxiliares de saúde bucal trabalhavam na área há mais de cinco anos e 12 (57,14\%) odontólogos há cerca de 20 anos.

Os dados apresentados na Tabela 1 mostram o conhecimento dos profissionais de saúde bucal sobre a classificação de produtos para saúde em produtos críticos, semicríticos e não críticos conforme Resolução da Diretoria Colegiada № 15/2012. Em geral a afirmativa com maior acerto pelos profissionais de saúde bucal foi em relação à definição de produto crítico por 20 (90,90\%) auxiliares de saúde bucal e 21 (100\%) odontólogos. 
Tabela 1 - Frequência das respostas na avaliação do conhecimento dos profissionais de saúde bucal sobre a classificação de produtos para saúde. São Carlos, 2014

\begin{tabular}{|c|c|c|c|c|c|c|}
\hline \multirow[t]{2}{*}{$\begin{array}{l}\text { Classificação de produtos } \\
\text { para saúde }\end{array}$} & \multicolumn{2}{|c|}{$\begin{array}{c}\text { Auxiliares de } \\
\text { Saúde Bucal } \\
n=22\end{array}$} & \multicolumn{2}{|c|}{$\begin{array}{l}\text { Odontólogos } \\
n=21\end{array}$} & \multicolumn{2}{|c|}{$\begin{array}{l}\text { Total } \\
n=43\end{array}$} \\
\hline & $\mathrm{N}$ & $\%$ & $\mathrm{~N}$ & $\%$ & $\mathrm{n}$ & $\%$ \\
\hline $\begin{array}{l}\text { Um instrumental } \\
\text { odontológico que penetra } \\
\text { os tecidos entrando em } \\
\text { contato com sangue é } \\
\text { considerado um produto } \\
\text { crítico e, portanto, deve ser } \\
\text { esterilizado. (C) }\end{array}$ & 20 & 90,90 & 21 & 100 & 41 & 95,34 \\
\hline $\begin{array}{l}\text { É considerado produto } \\
\text { crítico aquele utilizado em } \\
\text { qualquer procedimento } \\
\text { odontológico. (E) }\end{array}$ & 11 & 50,00 & 6 & 28,57 & 17 & 39,53 \\
\hline $\begin{array}{l}\text { Produto semicrítico é } \\
\text { aquele que entra em } \\
\text { contato com a pele não } \\
\text { íntegra ou com mucosa do } \\
\text { paciente e, portanto, requer } \\
\text { desinfecção de alto nível ou } \\
\text { esterilização para uso. (C) }\end{array}$ & 18 & 81,81 & 9 & 42,85 & 27 & 62,79 \\
\hline $\begin{array}{l}\text { Artigo não crítico é aquele } \\
\text { que entra em contato com } \\
\text { a pele íntegra e, portanto, } \\
\text { requer desinfecção de alto } \\
\text { nível ou esterilização para } \\
\text { uso. (E) }\end{array}$ & 7 & 31,81 & 5 & 23,80 & 12 & 27,90 \\
\hline $\begin{array}{l}\text { Artigo não crítico é aquele } \\
\text { que entra em contato } \\
\text { com a pele íntegra e, } \\
\text { portanto, requer a limpeza } \\
\text { entre o uso em diferentes } \\
\text { pacientes. (C) }\end{array}$ & 13 & 59,09 & 11 & 52,38 & 24 & 55,81 \\
\hline
\end{tabular}

Fonte: elaborada pelas autoras

A Tabela 2 apresenta os dados referentes ao conhecimento dos profissionais de saúde bucal sobre: esterilização, desinfecção, limpeza, recomendações e embalagens. 
Tabela 2 - Frequência de respostas na avaliação do conhecimento dos profissionais de saúde bucal sobre definições no processamento de produtos para saúde. São Carlos, 2014

\begin{tabular}{|c|c|c|c|c|c|c|}
\hline \multirow[t]{2}{*}{ Definiç̧̃es } & \multicolumn{2}{|c|}{$\begin{array}{c}\text { Auxiliares de } \\
\text { Saúde Bucal } \\
n=22\end{array}$} & \multicolumn{2}{|c|}{$\begin{array}{l}\text { Odontólogos } \\
n=21\end{array}$} & \multicolumn{2}{|c|}{$\begin{array}{l}\text { Total } \\
n=43\end{array}$} \\
\hline & $N$ & $\%$ & $\mathrm{~N}$ & $\%$ & $\mathrm{n}$ & $\%$ \\
\hline $\begin{array}{l}\text { A esterilização é o processo capaz de eliminar } \\
\text { todas as formas de vida microbiana, incluindo } \\
\text { os esporos bacterianos. (C) }\end{array}$ & 18 & 81,81 & 18 & 85,71 & 36 & 83,72 \\
\hline $\begin{array}{l}\text { A desinfecção é processo que elimina } \\
\text { microrganismos em objetos inanimados, } \\
\text { exceto os esporos. (C) }\end{array}$ & 8 & 36,36 & 11 & 52,38 & 19 & 44,18 \\
\hline $\begin{array}{l}\text { Limpeza é remoção de sujidades orgânicas e } \\
\text { inorgânicas, com redução da carga microbiana } \\
\text { presente nos produtos para saúde, utilizando- } \\
\text { se água, detergentes, produtos e acessórios de } \\
\text { limpeza, por meio de ação mecânica (manual } \\
\text { ou automatizada), atuando em superfícies } \\
\text { internas (lúmen) e externas, de forma a tornar } \\
\text { o produto seguro para manuseio e preparado } \\
\text { para desinfecção ou esterilização. (C) }\end{array}$ & 19 & 86,36 & 18 & 85,71 & 37 & 86,04 \\
\hline $\begin{array}{l}\text { Limpeza é um processo que visa à remoção de } \\
\text { sujidade, somente em artigos não críticos. (E) }\end{array}$ & 2 & 9,09 & 6 & 28,57 & 8 & 18,60 \\
\hline $\begin{array}{l}\text { A limpeza é uma etapa desnecessária } \\
\text { para artigos críticos, uma vez que ele será } \\
\text { esterilizado. (E) }\end{array}$ & 0 & 0 & 2 & 9,52 & 2 & 4,65 \\
\hline $\begin{array}{l}\text { Para a esterilização adequada de um instrumental } \\
\text { odontológico, esta deve seguir obrigatoriamente } \\
\text { as etapas de limpeza, secagem, embalagem e } \\
\text { esterilização por autoclave ou estufa. (E) }\end{array}$ & 15 & 68,18 & 13 & 61,90 & 28 & 65,11 \\
\hline $\begin{array}{l}\text { Para a esterilização adequada de um } \\
\text { instrumental odontológico, esta deve seguir, } \\
\text { obrigatoriamente, as etapas de limpeza, } \\
\text { secagem, embalagem e esterilização por } \\
\text { autoclave. (C) }\end{array}$ & 18 & 81,81 & 16 & 76,19 & 34 & 79,06 \\
\hline $\begin{array}{l}\text { É recomendada, mas não obrigatória, a } \\
\text { realização de teste para avaliar o desempenho } \\
\text { do sistema de remoção de ar (Bowie \& Dick) } \\
\text { da autoclave assistida por bomba de vácuo, no } \\
\text { primeiro ciclo do dia. (E) }\end{array}$ & 5 & 22,72 & 6 & 28,57 & 11 & 25,58 \\
\hline $\begin{array}{l}\text { Não é permitido o uso de embalagens de } \\
\text { papel kraft, papel toalha, papel manilha, papel } \\
\text { jornal e lâminas de alumínio, assim como } \\
\text { as embalagens tipo envelope de plástico } \\
\text { transparente não destinadas ao uso em } \\
\text { equipamentos de esterilização.(C) }\end{array}$ & 12 & 54,54 & 16 & 76,19 & 28 & 65,11 \\
\hline
\end{tabular}

Fonte: elaborada pelas autoras 
Os resultados referentes ao conhecimento da equipe de saúde bucal sobre o tipo de embalagem utilizada no processamento são apresentados na Tabela 3.

Tabela 3 - Frequência de respostas na avaliação do conhecimento dos profissionais de saúde bucal sobre embalagem de produtos para saúde. São Carlos, 2014

\begin{tabular}{|c|c|c|c|c|c|c|}
\hline \multirow[t]{2}{*}{ Definições } & \multicolumn{2}{|c|}{$\begin{array}{l}\text { Auxiliares de } \\
\text { Saúde Bucal } \\
\quad \mathrm{N}=22\end{array}$} & \multicolumn{2}{|c|}{$\begin{array}{l}\text { Odontólogos } \\
\qquad \mathbf{N}=\mathbf{2 1}\end{array}$} & \multicolumn{2}{|c|}{$\begin{array}{l}\text { Total } \\
\mathrm{N}=43\end{array}$} \\
\hline & $\mathrm{N}$ & $\%$ & $\mathrm{~N}$ & $\%$ & $\mathrm{~N}$ & \\
\hline $\begin{array}{l}\text { Não é permitido o uso de embalagens de } \\
\text { papel kraft, papel toalha, papel manilha, } \\
\text { papel jornal e lâminas de alumínio, assim } \\
\text { como as embalagens tipo envelope de } \\
\text { plástico transparente. (C) }\end{array}$ & 5 & 22,72 & 9 & 42,85 & 14 & 32,55 \\
\hline $\begin{array}{l}\text { É permitido o uso de embalagens de papel } \\
\text { kraft para a esterilização de produtos para } \\
\text { saúde. (E) }\end{array}$ & 8 & 36,36 & 8 & 38,09 & 16 & 37,20 \\
\hline $\begin{array}{l}\text { Não é obrigatória a identificação dos } \\
\text { produtos para saúde nas embalagens. (E) }\end{array}$ & 0 & 0 & 1 & 4,76 & 1 & 2,32 \\
\hline $\begin{array}{l}\text { O uso de embalagens torna desnecessária a } \\
\text { higienização das mãos antes de descarregar } \\
\text { a autoclave. (E) }\end{array}$ & 1 & 4,54 & 2 & 9,52 & 3 & 6,97 \\
\hline $\begin{array}{l}\text { Apenas caixas metálicas com furos devem } \\
\text { ser empregadas para a esterilização de } \\
\text { produtos. (C) }\end{array}$ & 9 & 40,90 & 6 & 28,57 & 15 & 34,88 \\
\hline $\begin{array}{l}\text { A manipulação dos produtos esterilizados } \\
\text { deve ser realizada de forma asséptica. (C) }\end{array}$ & 14 & 63,63 & 13 & 61,90 & 27 & 62,79 \\
\hline $\begin{array}{l}\text { As embalagens utilizadas para a esterilização } \\
\text { de produtos para saúde devem ser } \\
\text { regulamentas para este fim pela ANVISA. (C) }\end{array}$ & 17 & 77,27 & 19 & 90,47 & 36 & 83,72 \\
\hline
\end{tabular}

Fonte: elaborada pelas autoras

\section{DISCUSSÃO}

Para que o processamento de produtos para saúde ocorra de forma adequada, torna-se importante que os profissionais dominem o conhecimento da classificação dos produtos para compreender o processo de esterilização.

Neste estudo, 19 (44,18\%) dos profissionais detêm o conhecimento correto da definição de desinfecção, esterilização (Tabela 2), fato perceptível também ao analisar 
a Tabela 1, o que possibilita relacionar o conhecimento da definição com a classificação dos produtos para saúde. O processamento de produtos requer, além da capacidade operacional para realização, a expertise necessária dos profissionais envolvidos. ${ }^{4}$

Estudo realizado em Centro Cirúrgico e Central de Materiais e Esterilização de um hospital público de Porto Alegre - RS mostrou que os profissionais de enfermagem não souberam descrever as etapas e os tipos de embalagens utilizados para esterilização e armazenamento do material cirúrgico. ${ }^{12}$

Em um curso de Odontologia em Belo Horizonte - MG, estudo sobre o controle de infecção cruzada, observou-se que 119 (59,2\%) estudantes negligenciam a desinfecção previamente à lavagem dos instrumentais contaminados. ${ }^{13}$

Em contrapartida com o encontrado na literatura, neste estudo o conhecimento dos profissionais de saúde bucal sobre as etapas de processamento foi satisfatório. Observa-se que a maioria tinha conhecimento sobre as definições de limpeza, 37 (86,04\%), esterilização, 36 (83,72\%), e tipo de embalagem, 36 (83,72\%).

Em estudo realizado em unidades básicas de saúde, encontrou-se deficiência no conhecimento dos profissionais e práticas inadequadas, tais como falta de produtos apropriados para a desinfecção, uso de produtos incorretos, inadequações no que se refere à validação das embalagens, lavagem e secagem inadequadas dos produtos para saúde, expurgo impróprio e higienização de mãos incorreta. ${ }^{14}$

Encontrou-se neste estudo que a maioria dos profissionais participantes do estudo, 15 (68,18\%) auxiliares de saúde bucal e 13 (61,90\%) odontólogos, afirmaram que a esterilização pode ser realizada por autoclave ou estufa (Tabela 2), mostrando a necessidade de atualização, uma vez que o uso de estufas pode comprometer a eficácia do processamento de esterilização dos produtos para saúde, interferindo em sua qualidade. De acordo com a RDC $n^{\circ} 15$, não é permitido o uso de estufas para a esterilização de produtos para saúde. ${ }^{11}$

Estudo realizado em consultórios odontológicos da rede pública do município de Porto Velho-RO avaliou a qualidade do processo de esterilização e detectou falhas nos procedimentos de rotina, como: utilização incorreta das relações tempo versus temperatura, falta de utilização de termômetros e do monitoramento dos equipamentos, entre outros. No monitoramento das estufas, $20 \%$ apresentaram resultados positivos, indicando falha no processo de esterilização. ${ }^{9}$

Ainda sobre os recursos materiais, neste estudo 11 (25,58\%) profissionais de saúde bucal equivocaram-se ao afirmar ser correta a não obrigatoriedade da realização de teste para avaliar o desempenho do sistema da autoclave (Tabela 2). Destaca-se um estudo realizado em 44 hospitais no interior do estado de Goiás, que mostrou que em apenas um (2,27\%) hospital realizavam-se os controles físico, químico e biológico, simultaneamente. Ainda, em $36(83,8 \%)$ hospitais eram utilizados apenas o controle químico. ${ }^{15}$ De acordo com a RDC $n^{\circ} 15$, é obrigatória a realização de teste para avaliar 
o desempenho do sistema de remoção de ar (Bowie \& Dick) da autoclave assistida por bomba de vácuo no primeiro ciclo do dia. ${ }^{11}$

Assim, para evitar falhas no processo de esterilização em autoclaves, o monitoramento deve ser realizado através da avaliação de parâmetros físicos, químicos e biológicos, sendo imperativo o uso de indicadores, que são instrumentos de extrema importância para adequação de processos internos de trabalho, salientando que a coleta e os resultados sejam tratados rigorosamente..$^{11-16}$

Estudo realizado em um município no estado de Alagoas encontrou que em 21 (95,45\%) Unidades Básica de Saúde (UBS) a monitorização visual do produto para saúde era realizada por meio de fita termossensível, enquanto o controle biológico era realizado em somente seis $(27,27 \%)$ UBS. ${ }^{17}$

No presente estudo, as afirmativas com maiores acertos sobre embalagens foram referentes à obrigatoriedade da regularização junto à Agência Nacional de Vigilância Sanitária (Anvisa) 83,72\% e sobre a manipulação destas ser realizada de forma asséptica $62,79 \%$ (Tabela 3). Dentre as boas práticas de controle de infecção, sobre o manuseio dos produtos esterilizados, conclui-se que a manipulação dos invólucros é uma variável importante na recontaminação dos materiais por bactérias, por ser a principal via de transmissão desses microrganismos. ${ }^{18-19}$

Dessa forma, neste estudo, os profissionais de saúde bucal demonstraram estar cientes da importância da higienização das mãos ao manusear o produto esterilizado, sendo essa ação de extrema importância para a prevenção e controle de infecções. ${ }^{20}$ Um estudo no nível municipal de Blumenau-SC apontou que $35(83,3)$ auxiliares de saúde bucal reconheciam o controle de infecção em odontologia como parte da temática de organização do ambiente de trabalho. O controle de IRAS em saúde bucal faz parte do processo de trabalho e é de competência dos profissionais envolvidos neste. ${ }^{21}$

Em contrapartida, um total de 16 (37,20\%) profissionais de saúde bucal afirmaram, segundo sua percepção, ser correto o uso de papel Kraft para esterilização de produtos para saúde (Tabela 3). De acordo com a RDC $n^{0} 15^{11}$, não é permitido o uso de embalagens de papel Kraft, papel-toalha, papel-manilha, papel-jornal e lâminas de alumínio, assim como as embalagens tipo envelope de plástico transparente não destinadas para o uso em equipamentos de esterilização. ${ }^{11}$

No presente estudo, 17 (77,27\%) auxiliares de saúde bucal e $18(85,71 \%)$ odontólogos apontam para a ausência de protocolo em sua rotina de trabalho. Ressalta-se que a ausência de protocolo dificulta a padronização do processamento de produtos, contribuindo para possíveis falhas, pois cada profissional pode realizar o processo segundo suas próprias interpretações. Cada etapa do processamento dos produtos para saúde deve seguir um Procedimento Operacional Padrão - POP elaborado com base em referencial científico atualizado e normas vigentes, devendo 
ser amplamente divulgado e disponível para consulta. ${ }^{11} \mathrm{Em}$ um estudo realizado em Picos-PI, 89\% das 19 unidades básicas de saúde estudadas não possuíam protocolos para a realização do processo de esterilização. ${ }^{22}$

Estudo realizado sobre o controle de infecções na odontologia do sistema prisional do estado brasileiro do Pará demonstrou que há deficiências graves nos procedimentos de prevenção e controle de infecção, como ausência de treinamento dos profissionais sobre o controle de IRAS. Foram avaliadas 11 clínicas de prisão, onde a lavagem manual de produtos para saúde sem uso de substâncias adequadas e esterilização por estufas sem o monitoramento do processo eram os principais métodos de descontaminação utilizados. ${ }^{23}$

Espera-se que os resultados deste estudo estimulem a realização de ações educativas e de vigilância para uma prática segura de processamento de produtos para a saúde executada pela equipe de saúde bucal da APS, além de trazer à tona a necessidade de ampliar essa discussão na formação dos profissionais e na realização de novas pesquisas na temática.

Como limitação deste estudo, destaca-se a dificuldade em conseguir maior participação dos profissionais da equipe de saúde bucal, interferindo na qualidade da amostra, bem como a realização em apenas um munícipio.

\section{CONCLUSÃO}

Os resultados do presente estudo indicam que os profissionais de saúde bucal entrevistados ainda possuem lacunas de conhecimentos sobre o processo de esterilização, bem como sobre a definição e uso do monitoramento através de indicadores de esterilização. Assim, as deficiências de conhecimento apontadas podem comprometer a qualidade do processamento de produtos à saúde e contribuir para o aumento do risco de IRAS.

Acredita-se que estudos como esse contribuam apontando temas para intervenções educativas futuras necessárias para as equipes. A produção de evidências científicas direciona as decisões a serem tomadas visando a melhorias na qualidade da assistência à saúde.

\section{REFERÊNCIAS}

1. Brasil. Ministério da Saúde. Secretaria de Atenção à Saúde. Departamento de Atenção Básica. Saúde Bucal. Brasília: Ministério da Saúde; 2008. 92 p.

2. Padoveze MC, Figueiredo RM. O papel da Atenção Primária na prevenção de Infecções Relacionadas à Assistência à Saúde. Rev esc enferm USP [Internet]. 2014 [acesso em 2019 dez 18]; 48(6):1137-44. Disponível em: <http://www.scielo.br/ pdf/reeusp/v48n6/pt_0080-6234-reeusp-48-06-1137.pdf $>$. 
3. Passos IBD, Padoveze MC, Roseira CE, Figueiredo RM. Adaptation and validation of indicators concerning the sterilization process of supplies in Primary Health Care services. Rev Latino-Am Enfermagem. [Internet]. 2015 [acesso em $2019 \mathrm{dez}$ 18]; 23(1):148-54. Disponível em: <http://www.scielo.br/scielo.php?script=sci_ arttext\&pid=S010411692015000100148>.

4. Padoveze $M C$, Fortaleza $C M C B$. Infecções relacionadas à assistência à saúde: desafios para a saúde pública no Brasil. Rev Saúde Pública [Internet]. 2014 [acesso em 2019 dez 16]; 48(6):9951001. Disponível em: <http://www.scielo.br/scielo.php?script=sci_ arttext\&pid=S003489102014000600995\&lng=en\&nrm=iso\&tlng=en>.

5. Reis WG, Scherer MDA, Carcereri DL. O trabalho do Cirurgião-Dentista na Atenção Primária à Saúde: entre o prescrito e o real. Saúde debate [Internet]. 2015 [acesso em 2019 dez 16]; 39(104):56-64. Disponível em: <http://www.scielo.br/scielo. php?script=sci_arttext\&pid=S010311042015000100056\&lng=en\&nrm=iso\&tlng=pt $>$.

6. Roseira CE, Silva DM, Passos IPBD, Orlandi FS, Padoveze MC, Figueiredo RM. Diagnóstico de conformidade do processamento de produtos para saúde na Atenção Primária à Saúde. Rev Latino-Am. Enfermagem [Internet]. 2016 [acesso em 2019 dez 18]; 24:e2820. Disponível em: <http://www.scielo.br/scielo. php?pid=S010411692016000100426\&script=sci_arttext\&tlng=pt $>$.

7. Brasil. Ministério da Saúde. Secretaria de Políticas de Saúde. Coordenação Nacional de DST e AIDS. Controle de infecções e a prática odontológica em tempos de AIDS: manual de condutas. Brasília; 2000.

8. Arantes DC, Hage CA, Nascimento LS, Pontes FSC. Biossegurança aplicada à Odontologia na Universidade Federal do Pará, Cidade de Belém, Estado do Pará, Brasil. Rev Pan-Amaz Saude [Internet]. 2015 [acesso em 2019 dez 16]; 6(1):11-8. Disponível em: <http://scielo.iec.gov.br/scielo.php?script=sci_ arttext\&pid=S217662232015000100002\&lng=pt>.

9. Dias AGA, Santos SSF, Nardo AO, Leao M. Esterilização no serviço público odontológico. ClipeOdonto 2014 [acesso em 2019 dez 19); 6(1):2-10. Disponível em: <http://periodicos.unitau.br/ojs/index.php/clipeodonto/article/view/1697/1391>.

10. Pimentel MJ, Filho MMVB, Santos JP, Rosa MRD. Biossegurança: comportamento dos alunos de Odontologia em relação ao controle de infeç̧ão cruzada. Cad. saúde colet. [Internet]. 2012 [acesso em 2019 dez 18]; 20(4):525-32. Disponível em: <http://www.scielo.br/pdf/cadsc/v20n4/v20n4a17.pdf>.

11. Brasil. Ministério da Saúde. Resolução - RDC n. 15, de 15 de Março de 2012. Dispõe sobre requisitos de boas práticas para o processamento de produtos para saúde e dá outras providências. 2012.

12. Ouriques $\mathrm{CM}$, Machado MÉ. Enfermagem no processo de esterilização de materiais. Texto contexto - enferm. [Internet]. 2013 [acesso em 2019 dez 16]; 22(3 ):695-703. Disponível em: <http://www.scielo.br/pdf/tce/v22n3/v22n3a16. pdf>. 
13. Zocratto K, Silveira A, Arantes D, Borges L. Conduta dos estudantes na clínica odontológica integrada em relação às normas de controle de infecção e biossegurança. RFO [Internet]. 2016 [acesso em 2019 dez 16]; 21(2). Disponível em: <http://www.seer.upf.br/index.php/rfo/article/view/6112>.

14. Fontana R, Strehlow B, Rosa A, Freitas C. A prática do processamento de artigos em unidades básicas. VD [Internet].2016 [acesso em 2019 dez 16]; 4(1):45-1. Disponível em: <https://visaemdebate.incqs.fiocruz.br/index.php/visaemdebate/article/ view/530>.

15. Tipple AFV, Pires FV, Guadagnin SVT, Melo DS. O monitoramento de processos físicos de esterilização em hospitais do interior do estado de Goiás. Rev. esc. enferm. USP [Internet]. 2011 [acesso em 2015 ago 29]; 45(3): 751-7. Disponível em: <http://www.scielo.br/scielo.php?script=sci_ arttext\&pid=S008062342011000300029\&lng=en>.

16. Fusco SFB, Spiri WC. Analysis of quality indicators of central sterile supply departments at accredited public hospitals. Texto contexto - enferm. [Internet]. 2014 [acesso em 2017 maio 3]; 23( 2 ): 42633. Disponível em: <http://www.scielo.br/scielo.php?script=sci_ arttext\&pid=S010407072014000200426\&lng=en>.

17. Vital JS, Lins TH, Veríssimo RCS, Souza EMS. Estrutura física de centro de material e esterilização em unidades de atenção básica de saúde. Rev enferm UFPE on line. 2014 [acesso em 2019 dez 18]; 8(5):1192-200. Disponível em: <https://periodicos. ufpe.br/revistas/revistaenfermagem/article/download/9799/9963>.

18. Schwaab G, Jacoby AM, Lunkes JT, Ascari RA, Lautert L. Esterilização de produtos para saúde em serviços públicos. Rev. enferm. UFPE on line. 2016 [acesso em 2019 dez 18]; 10(12), 4591-98. Disponível em: <https://periodicos.ufpe.br/revistas/ revistaenfermagem/article/viewFile/11527/1342>.

19. Nunes MBS, Fassarella CS, Souza PA de, Meneses R de O, Silva MVG da, Luna AA. Condições de temperatura e umidade na sala de distribuição e armazenamento dos produtos para saúde. Rev Enferm Atual [Internet]. 2019 [acesso em 2019 dez 16]; 87(Edição Esp). Disponível em: <https://revistaenfermagematual.com.br/index.php/ revista/article/view/260>.

20. Baraldi MM, Padoveze MC. Higienização das Mãos: a evolução e o atual "Estado da Arte”. J Infect Control [Internet]. 2015 [acesso em 2019 dez 16]; 4(3):1-2 Disponível em: <http://jic-abih.com.br/index.php/jic/article/view/114>.

21. Warmling CM , Cipriani CR. Perfil de auxiliares e técnicos em saúde bucal que atuam no sistema único de saúde. Rev APS [Internet]. 2017 [acesso em 2019 dez 16]; 19(4): 592-601.Disponível em: <https://aps.ufjf.emnuvens.com.br/aps/article/ view/2560>.

22. Freitas TS, Quirino GS. Esterilização em unidades básicas de saúde no município de Picos-PI. SANARE [Internet]. 2013 [acesso em 2019 dez 19]; 9(10):57-63. Disponível em <https://sanare.emnuvens.com.br/sanare/article/view/256/229>. 
23. Lima CMA, Smith AJ, Silva ASF, Flório FM, Zanin L. Infection prevention and control in dental surgeries in the Pará state prison system in Brazil. Am J Infect Control [Internet]. 2016 [acesso em 2019 dez 16];44:1404-5. Disponível em: <https://www. ajicjournal.org/article/S0196-6553(16)30296-6/fulltext>.

Submissão: maio de 2017.

Aprovação: janeiro de 2020. 